\title{
Nano-Dot Markers for Electron Tomography Formed by Electron Beam- Induced Deposition: Nanoparticle Agglomerates Application
}

\author{
Misa Hayashida ${ }^{1}$, Marek Malac ${ }^{2,3}$, Michael Bergen ${ }^{2}$, Peng $\mathrm{Li}^{2}$
}

1. National Institute of Advanced Industrial Science and Technology (AIST), 1-1-1, Higashi, Tsukuba, Ibaraki 305-8565, Japan

2. National Institute of Nanotechnology, 11421 Saskatchewan Drive, Edmonton, Canada

3. Department of Physics, University of Alberta, T6G 2E1, Edmonton, Canada

Electron tomography is a method employed in a transmission electron microscope (TEM) to reconstruct a three-dimensional (3D) volume from a series of images acquired at suitable tilt increments. An easy, accurate and preferably fully automated alignment of the individual images in the series is critical to obtain good quality 3D reconstruction of the sample. Fiducial markers, usually gold nanoparticles placed at random positions of the sample from a suspension, are often used for tomography of biological samples. For precise alignment, markers must be uniformly dispersed over the observed region of the specimen. However, it is difficult to obtain even dispersion because the colloidal gold nanoparticles are usually dense materials [1]. If dense, high contrast, gold nanoparticles are in close proximity or in contact with the specimen, they introduce artifacts in the reconstructed 3D images. However, when fiducial markers are not present precise, alignment of individual images of the series cannot be carried out.

In a previous study, we reported the use of a helium-ion microscope (HIM) equipped with a tungsten carbonyl $\left(\mathrm{W}(\mathrm{CO})_{6}\right)$ gas injection system (GIS) to form tungsten nanodots $[2,3]$. We demonstrated for the first time, the suitability of fabricated nanodots with $\sim 10 \mathrm{~nm}$ diameter as fiducial markers for precise alignment of a TEM tomographic tilt series of a rod-shaped specimen. To make the method accessible to a broad research community, the nanodots marker fabrication had to be transferred from HIM to instruments that are readily accessible. Here we report fabrication of nanodot fiducial markers by electron beam-induced deposition of tungsten in a standard scanning electron microscope (SEM) [4] equipped with a GIS system.

Two samples were prepared to demonstrate the nanodots markers suitability for precise alignment of electron tomography tilt series: a sample composed of $\mathrm{TiO}_{2}$ nanoparticles (NPs) with lateral dimensions $\sim 50 \mathrm{~nm}$ and a sample of a few-layer thick ordered array of silver NPs with sub-5 nm diameter.

Rod-shaped specimens were fabricated by focused ion beam (FIB) milling of cube-shaped samples in a Hitachi NB 5000 dual beam (FIB/SEM) instrument. The diameters of rod-shaped specimens with $\mathrm{TiO}_{2}$ and Ag NPs were about $300 \mathrm{~nm}$ and $250 \mathrm{~nm}$ respectively. Nano-dot markers were fabricated onto the rod shaped specimen using electron beam induced-deposition of tungsten from $\mathrm{W}(\mathrm{CO})_{6}$ precursor.

Annular dark field (ADF) scanning TEM (STEM) was used to image the $\mathrm{TiO}_{2} \mathrm{NPs}$ and conventional TEM was used for the Ag NPs to demonstrate suitability of the nano-dot markers for each method. The tilt step was $3^{\circ}$ over the entire $0^{\circ}$ to $180^{\circ}$ tilt range for both samples. Hitachi HF $3300 \mathrm{TEM} / \mathrm{STEM}$ controlled by Maestro, a Matlab-based computer control system was used for both experiments [5].

Figure 1 shows a section of the reconstructed volume of the TiO2 NP sample in a plane that includes the long axis (tilt axis) of the rod-shaped sample (b) and a plane perpendicular to the long axis of the sample rod (the tilt axis) in c) to f). While the NPs can be clearly distinguished in areas where they are not touching, the interface in areas where NPs are touching is hard to detect the individual NPs as they may be fused together. Since the NPs fusing appears to be uniform throughout the cross section rather than near the sample edges (Fig. 1b, e and f) the NPs fusing is unlikely to be a result of the rod 
fabrication in the FIB instrument. Figure 2 shows the second application example of the nano-dot markers to a difficult test sample. In this case, sub-5 nm diameter Ag nanoparticles were deposited in a regular array on a Si wafer. The Ag NPs' boundary appears sharp and the individual Ag NPs are clearly visible in a cross section of the reconstructed volume, which indicates good alignment using the e-beam fabricated fiducial nano- dot markers.

[1] M. C. Lawrence, in: Frank, J. (Ed.), Electron Tomography. Plenum Press, New York, (1992) 197.

[2] M.Hayashida, T. Iijima, T. Fujimoto and S. Ogawa, Micron 43 (2012), 992-995.

[3] M. Hayashida, T. Iijima, M. Tsukahara and S. Ogawa, Micron 50 (2013), 29-34.

[4] M. Hayashida et. al, (Submitted to Ultramicroscopy)

[5] M. Bergen et. al. Microscopy and Microanalysis 19 (S2), 1394-1395 (2013).

(a)

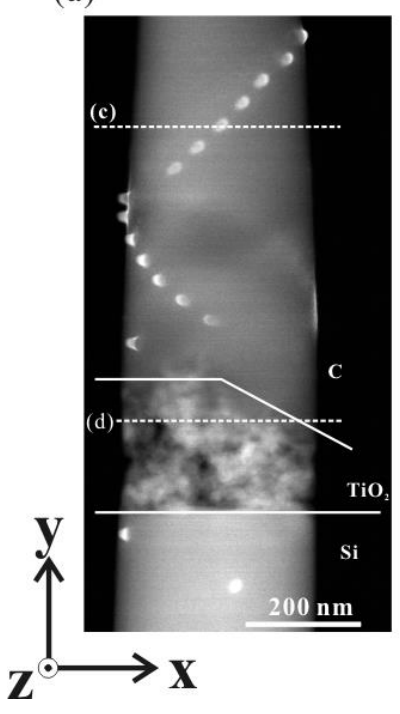

(a)

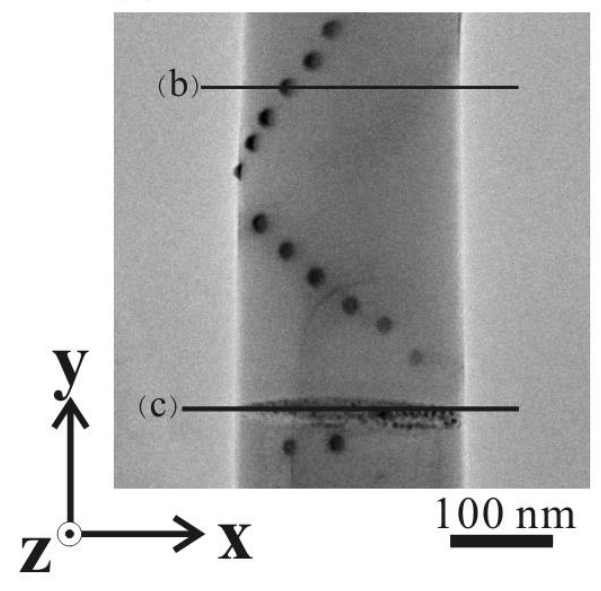

(b)

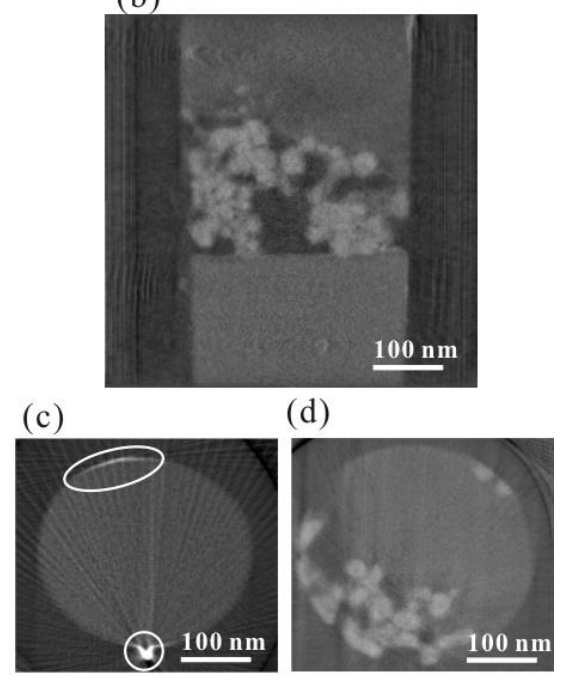

Figure 1. Electron tomographic observation of $\mathrm{TiO}_{2}$ NPs on a $\mathrm{Si}$ substrate. a) An experimental ADF STEM projected image. The sample regions are (starting from the top): amorphous carbon with nano-dot markers, $\mathrm{TiO}_{2} \mathrm{NPs}$, Si wafer substrate. The scale bar is $200 \mathrm{~nm}$. b) A cross section from the reconstructed volume in a plane that includes the tilt axis. (c)a cross section of reconstructed volume of nano-dot markers in a plane perpendicular to the tilt axis. The plane of the cross section is marked in a). (d) a cross section of reconstructed volume of $\mathrm{TiO}_{2}$ NPs in a plane perpendicular to the tilt axis.

(b)

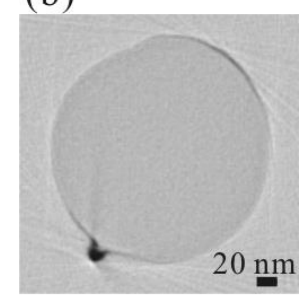

(c)

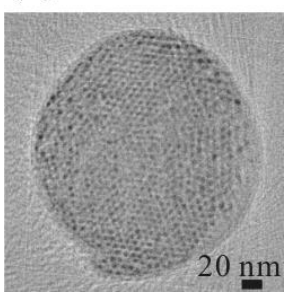

Figure 2. Electron tomographic observation of $\mathrm{Ag}$ NPs on Si substrate. a) Bright field TEM image of the entire specimen. The regions are (starting from the top): amorphous carbon with nano-dot markers, ordered array of $\mathrm{Ag}$ NPs and a $\mathrm{Si}$ wafer substrate.

b) A cross section of reconstructed volume of nano-dot markers in a plane perpendicular to the tilt axis. The plane of the cross section is marked in a). (c) a cross section of reconstructed volume of Ag NPs in a plane perpendicular to the tilt axis. 